\title{
The involvement of FoxO in cell survival and chemosensitivity mediated by Mirk/Dyrk1B in ovarian cancer
}

\author{
JINGCHUN GAO $^{1 *}$, XIANGJUN YANG ${ }^{2 *}$, PING YIN $^{3}$, WENFENG HU ${ }^{2}$, \\ HONGFENG LIAO ${ }^{3}$, ZHIHUI MIAO ${ }^{2}, \mathrm{CHAO} \mathrm{PAN}^{3}$ and $\mathrm{NALI}^{2}$ \\ ${ }^{1}$ Department of Obstetrics and Gynecology, First Affiliated Hospital, Dalian Medical University, Dalian, \\ Liaoning 116011; Departments of ${ }^{2}$ Obstetrics and Gynecology, ${ }^{3}$ Pathology, Zhongshan Hospital \\ Xiamen University, Xiamen, Fujian 361004, P.R. China
}

Received September 21, 2011; Accepted November 18, 2011

DOI: $10.3892 /$ ijo.2011.1293

\begin{abstract}
Minibrain-related kinase (Mirk) is a serine/threonine kinase which is also known as the dual specificity tyrosinephosphorylation-regulated kinase 1B (Dyrk1B). It is known that Dyrk1A, the closest family member to Mirk/Dyrk1B can mediate cellular localization of mammalian forkhead subclass $\mathrm{O}$ (FoxO1), a transcription factor, although the effect of Mirk/ Dyrk1B on FoxO factors remains to be defined. In this study, we showed that Mirk/Dyrk1B protein was overexpressed in 5 of 8 ovarian cancer cell lines and negatively correlated with the protein level of FoxO factors (FoxO1+FoxO3A). Knockdown of Mirk by small interfering RNA (siRNA) resulted in cell apoptosis and sensitized cells to cisplatin accompanied by nuclear translocation of FoxO1 and/or FoxO3A as well as increased Bim, TRADD, cleaved caspase-3 and PARP. Furthermore, combined siRNAs of Mirk with FoxO1 and/or FoxO3A led to fewer apoptotic cells and cisplatin sensitivity compared to Mirk siRNA alone, suggesting that FoxO is involved in Mirkmediated cell survival and chemosensitivity of ovarian cancer. Taken together, Mirk/Dyrk1B plays an important role in ovarian cancer cell survival through modulating FoxO translocation and may be a novel therapeutic target for ovarian cancer.
\end{abstract}

\section{Introduction}

Ovarian cancer is the fourth most common cause of cancer deaths in women exceeded only by breast, colon and lung

Correspondence to: Dr Jingchun Gao, Department of Obstetrics and Gynecology, First Affiliated Hospital, Dalian Medical University, Zhongshan Road 222, Dalian, Liaoning 116011, P.R. China

E-mail: jingchun_gao@yahoo.com

Dr Ping Yin, Department of Pathology, Zhongshan Hospital Xiamen University, Hubin Nan Road 201-209, Xiamen, Fujian 361004, P.R. China

E-mail: yinping20022002@yahoo.com.cn

*Contributed equally

Key words: minibrain-related kinase/dual specificity tyrosinephosphorylation-regulated kinase $1 \mathrm{~B}$, forkhead subclass $\mathrm{O}$, apoptosis, ovarian cancer malignancies with the majority of cases being diagnosed after the disease has become metastatic, and the 5-year survival is about $40 \%$ (1). Although the chemotherapeutic agents, such as cisplatin, carboplatin and paclitaxel have been known to be effective against ovarian carcinomas, the efficacy of which is limited by intrinsic or acquired chemoresistance in residual cells. Therefore, it is warranted to explore new therapeutic target for further treatment and reducing recurrence of the disease.

Minibrain-related kinase (Mirk) is a serine/threonine kinase which is also known as the dual specificity tyrosine-phosphorylation-regulated kinase 1B (Dyrk1B). Mirk/Dyrk1B is one of members of Dyrk family which have the ability to auto-phosphorylate on tyrosine and then phosphorylate other substrates on serine and threonine (2); therefore, they are categorized as dual function kinases. Mirk/Dyrk1B is expressed in few normal tissues, but in many types of human cancer (3), such as sarcomas $(4,5)$, pancreatic and colon carcinomas (6), and cervical cancer (7). Our recent study also found Mirk/Dyrk1B was overexpressed in a wide spectrum of cell lines and tumor specimens of lung cancer (8). Furthermore, the knockdown Mirk/Dyrk1B by small interfering RNA (siRNA) induced cell apoptosis and increased sensitivity of human cancer cells to conventional chemotherapeutics in vitro $(5,6,8)$. Our previous results also showed Mirk/Dyrk1B function in an orthotopic mouse model (8). Moreover, a study in osteosarcoma demonstrates that the overall survival rate of patients is negatively correlated with the levels of Mirk/Dyrk1B protein expression (5). All of the above suggest that Mirk/Dyrk1B could serve as a novel therapeutic target and the overexpressed Mirk/Dyrk1B may be a diagnostic marker and survival factor for various types of human cancer.

The mammalian forkhead subclass $\mathrm{O}$ (FoxO) family members of transcriptional factors, such as FoxO1 (FHKR) and FoxO3a (FKHR-L1) are characterized by a distinctive forkhead DNA binding domain which function downstream of PI3K antagonist PTEN in cancer cells, inhibit cell cycle progression and promote cell death by modulating the expression of genes encoding apoptosis (9), growth regulatory proteins (10) and stress response $(11,12)$. The modulating mechanisms include: a) direct binding to the insulin response sequence (IRS) in gene promoters (e.g., apoptotic proteins Bim and fas ligand) and b) tethering to the other transcription factors (cell cycle regulators 
cyclin G2 and cyclin D1). The phosphorylation of FoxO factors by protein kinases, such as Akt, serum and glucocorticoid inducible kinase (SGK) leads to their translocation from the nucleus to the cytoplasm and loss of proapoptotic function due to inactivation $(13,14)$. Whereas, the unphosphorylated active forms of FoxO reside in the nucleus and induces cell death by up-regulation of apoptotic proteins, such as Bim, p27, TRADD (15-17) and repression of antiapoptotic molecule FLIP and Bcl-XL $(18,19)$. Furthermore, Dyrk1A, the closest family member to Mirk/Dyrk1B, has been found to phosphorylate FoxO1 at ser329, a novel in vivo phosphorylation site (20), and mediates cellular localization of FoxO1 in immortalized cells (21). More recently, the serine/threonine kinase Mirk/Dyrk1B has been thought to be a transcriptional co-activator which increases expression of a cohort of antioxidants in human cancer cells $(22,23)$. In addition, both FoxO1 and FoxO3a have been reported to be involved in cytotoxic stress and drugresistance induced by chemotherapeutics in ovarian cancers $(24,25)$. Taken together, we hypothesize that FoxO factors may be a novel downstream manner by which Mirk/Dyrk1B serves as an antiapoptotic factor and contribute to ovarian cancer cell survival.

Although a few studies show that Mirk/Dyrk1B mediates ovarian cancer cell survival, in particular for quiescent tumor cells, and depleting Mirk kinase increase cisplatin toxicity associated with higher level of reactive oxygen species (ROS) in ovarian cancer cells $(23,26)$, insufficient data regarding the effect of Mirk/Dyrk1B on human ovarian cancer cells are available, and the mechanisms involved remain unclear. In this study, we have identified that Mirk/Dyrk1B is overexpressed in a wide spectrum of ovarian cancer cell lines and primary tumors in which it is located in the cytoplasm. Mirk/Dyrk1Bmediated cell survival and chemosensitivity is correlated with expression and nuclear translocation of FoxO1 and/or FoxO3A in ovarian cancer.

\section{Materials and methods}

Antibodies. The rabbit polyclonal Dyrk1B antibody (C-term, AP7538b) was purchased from Abgent (San Diego, CA). Anti-Bim, anti-TRADD, and goat anti-mouse IgG horseradish peroxidase (HRP)-conjugated secondary antibody were purchased from Santa Cruz Biotechnology (Santa Cruz, CA). Anti-FKHR/FoxO1, anti-caspase-3, and anti-poly(ADP-ribose) polymerase (PARP) were purchased from Cell Signaling Technology (Danvers, MA). Anti-FKHR-L1/FoxO3a was purchased from Upstate (Lake Placid, NY). Alexa Fluor 594 $F\left(a b^{\prime}\right)$ fragment of goat anti-mouse IgG were purchased from Invitrogen (Eugene, OR). Anti- $\beta$-actin and donkey anti-rabbit IgG HRP-conjugated secondary antibody were purchased from Sigma (St. Louis, MO) and Amersham Biosciences (Piscataway, $\mathrm{NJ}$ ), respectively.

Cell lines and cell culture. Human ovarian cancer cell lines used were OV2008, OVCAR3, OVCAR5, SKOV3, MDAH2774, OVCAR10, OV1063, OVCAR8. The SKOV3 and OVCAR3 were purchased from American Type Culture Collection (Manassas, VA); others were gifts from Dr Jin Q. Cheng (H. Lee Moffitt Cancer Center and Research Institute, USA). All lines were maintained in DMEM supplemented with
$10 \%$ heat-inactivated $\left(56^{\circ} \mathrm{C}, 30 \mathrm{~min}\right)$ fetal bovine serum (FBS; Invitrogen, Grand Island, NY). Monolayer cultures were incubated at $37^{\circ} \mathrm{C}$ in a $95 \%$ humidified atmosphere air containing $5 \% \mathrm{CO}_{2}$.

Smallinterfering RNAtreatment.Cells werereverse-transfected with small interfering RNAs (siRNAs) using Lipofectamine 2000 transfection reagent (Invitrogen) according to the manufacturer's instructions. The Mirk/Dyrk1B, FoxO1 and FoxO3a siRNA duplexes as well as the corresponding non-specific control siRNA duplexes as described $(8,27)$ were supplied by Dharmacon and Ambion, respectively. After a 72-h incubation or at indicated time points, cells were harvested or trypsinized and replated for subsequent experiments.

Flow cytometry analysis. After 72-h treatment with siRNAs, cells were subjected to flow cytometry analyses of apoptosis. Apoptosis was assayed using Pharmingen (San Diego, CA) PE-conjugated monoclonal active caspase-3 antibody apoptosis kit without modification as described previously (8). A total of 10,000 cells per experimental condition were used for processing and analysis of fluorescence on Becton-Dickinson FACScan (BD, Franklin Lakes, NJ) using CellQuest software. Apoptosis of siRNA-transfected cells after 48-h exposure to the chemotherapeutic agent cisplatin (CDDP) was also detected by flow cytometry analysis.

Western blot analysis. Cells were washed twice with cold PBS and lysed with buffer A [10 mM Tris- $\mathrm{HCl}$ (pH 7.4), $1 \%$ Triton X-100, 0.1\% SDS, $150 \mathrm{mM} \mathrm{NaCl}, 1 \mathrm{mM}$ EDTA, $1 \mathrm{mM}$ dithiothreitol, $0.5 \mathrm{mM}$ phenylmethylsulfonyl fluoride, $10 \mu \mathrm{g} /$ $\mathrm{ml}$ leupeptin, $5 \mu \mathrm{g} / \mathrm{ml}$ aprotinin]. After incubation for $30 \mathrm{~min}$ on ice, the suspensions were centrifuged $(15,000 \mathrm{~g}$ for $30 \mathrm{~min})$. The supernatants were removed and stored at $-80^{\circ} \mathrm{C}$ until analysis using gel electrophoresis. The protein concentration was determined by Bio-Rad protein estimation assay according to the manufacturer's instructions. For Western blot analysis, $\sim 60-100 \mu \mathrm{g}$ of whole cell proteins were separated using $10 \%$ or $12 \%$ SDS-PAGE and transferred to nitrocellulose membranes. After blocking of the membranes with $10 \mathrm{mM}$ Tris- $\mathrm{HCl}$ (pH 7.4), $150 \mathrm{mM} \mathrm{NaCl}$, and $0.1 \%$ Tween- 20 containing $5 \%$ non-fat dry milk at room temperature for $60 \mathrm{~min}$, the membranes were incubated with indicated antibodies at $4^{\circ} \mathrm{C}$ overnight and then with the HRP-conjugated secondary anti-rabbit or anti-mouse antibodies at room temperature for $60 \mathrm{~min}$. Each protein was detected using the enhanced chemiluminescence (Amersham Biosciences) system. $\beta$-actin was used as an internal control.

Patients and tumor specimens. The primary human ovarian cancer specimens were obtained from 51 patients who underwent surgery without chemotherapy or radiation prior to resection at the First Affiliated Hospital of Dalian Medical University and Zhongshan Hospital Xiamen University between 1996 and 2010. Each sample contained at least $80 \%$ tumor cells, confirmed by microscopic examination. As control groups, the specimens obtained from 16 patients with ovarian benign tumor and 9 cases of non-neoplastic cyst were also examined. The clinicopathological aspects of all samples were listed in Table I. The study was approved by the Research Committee of 
Table I. Clinicopathological aspects and Mirk expression in patients.

\begin{tabular}{lc}
\hline Characteristic & No. of patients \\
\hline Total & 76 \\
Age (years) ${ }^{\mathrm{a}}$ & 47 \\
Histology (positive for Mirk) $^{\mathrm{b}}$ & \\
Cystadenocarcinomas & $51(38)$ \\
$\quad$ Serous & $38(31)$ \\
$\quad$ Mucinous & $13(7)$ \\
Cystadenomas & $16(6)^{\mathrm{c}}$ \\
$\quad$ Serous & $9(3)$ \\
$\quad$ Mucinous & $7(3)$ \\
$\quad$ Non-neoplastic cysts & $9(0)^{\mathrm{d}}$ \\
\hline
\end{tabular}

${ }^{\mathrm{a}}$ Median age was 47 years (range $=32-64$ ). ${ }^{\mathrm{b}}$ Comparison of the incidence of cytoplasmic Mirk expression between subgroups. ${ }^{c} \mathrm{P}<0.05$ and ${ }^{\mathrm{d}} \mathrm{P}<0.0001$ compared with cystadenocarcinomas.

the First Affiliated Hospital of Dalian Medical University and Zhongshan Hospital Xiamen University.

Immunostaining analysis of Mirk/DyrklB and FoxO. Immunohistochemistry staining using anti-Dyrk1B as the primary antibody. After antigen retrieval with citrate, the endogenous peroxidase activity was blocked by incubation with $0.3 \%$ hydrogen peroxide. Slides were incubated overnight with 1:50 primary antibody at $4^{\circ} \mathrm{C}$. Antigen-antibody complexes were detected by the avidin-biotin peroxidase method using $\mathrm{ABC}$ Kit (Vector Laboratories, Inc., Burlingame, CA) and DAB (Dako, Japan) reagents. Sections were counterstained with hematoxylin and viewed using a microscope (Zeiss, Tokyo, Japan). For immunofluorescent staining, cells were fixed with $4 \%$ paraformaldehyde for $20 \mathrm{~min}$ on ice. Cells were incubated in $1 \%$ bovine serum albumin (BSA) in PBS for 30 min. Primary antibody against FoxO1 or FoxO3a (1:100) was added in 1\% BSA/PBS for overnight at $4{ }^{\circ} \mathrm{C}$. After washing, cells were incubated with Alexa Fluor $594 \mathrm{~F}\left(\mathrm{ab}^{\prime}\right)$ fragment of goat anti-mouse IgG for $30 \mathrm{~min}$ at room temperature, and nuclei were then counterstained with DAPI allowing visualization of nuclei with a Leica Confocal Microscope System.

Statistical analysis. Each experiment was repeated three times. Data are presented as mean \pm SD. StatView 5.0 software was used for statistical analyses. Statistical comparison between control and experimental groups were performed using $\chi^{2}$ test (for incidence only) and Student's t-test. The correlations between Mirk expression and FoxO were analyzed by simple regression. Differences were considered to be statistically significant when $\mathrm{P}<0.05$.

\section{Results}

Mirk is widely overexpressed in ovarian cancer cells and correlates with FoxO expression. In this study, we first evalu-
$\mathbf{A}$

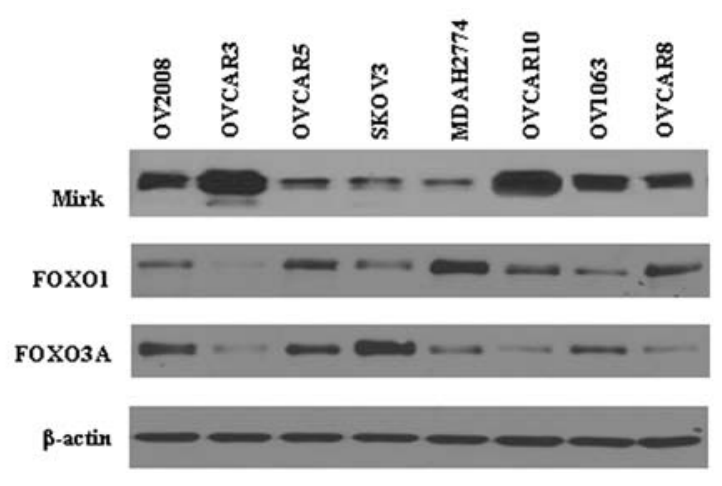

B

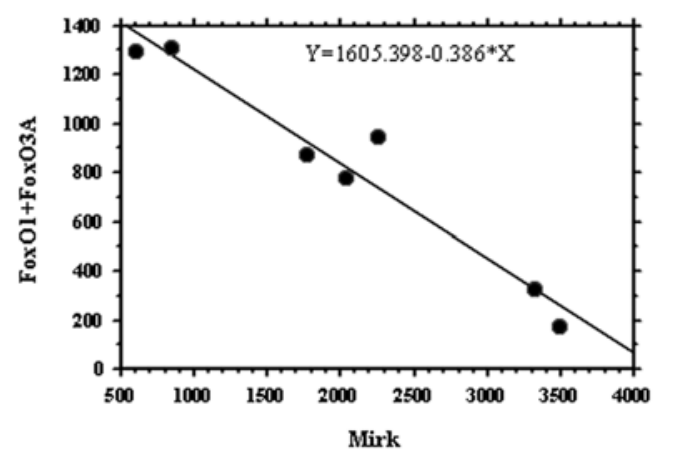

Figure 1. Mirk is overexpressed in a wide variety of ovarian cancer cell lines and correlated with FoxO expression. (A), Western blot analysis of Mirk (69 and $71 \mathrm{kDa})$, FoxO1 (70 kDa) and FoxO3A (97 kDa) in ovarian cancer cells. Equal loading and transfer were shown by repeat probing with $\beta$-actin $(42 \mathrm{kDa})$. (B), The correlation between the expression of Mirk protein and the total level of FoxO (FoxO1+FoxO3A) was analyzed by simple regression.

ated protein expression of Mirk in 8 human ovarian cancer cell lines. The 8 cell lines all expressed Mirk protein, 5 of them with high levels (Fig. 1A), which is consistent with the findings reported by Hu et al (26). Based on the hypothesis described above that the FoxO transcriptional factors may be involved in Mirk function in ovarian cancer, we further examined the expression of both FoxO1 and FoxO3A in the 8 cell lines (Fig. 1A). As shown in Fig. 1B, correlation appears to be negative between the expression of Mirk protein and the total level of FoxO (FoxO1+FoxO3A) in ovarian cancer cells $\left(\mathrm{R}^{2}=0.946\right.$ and $\left.\mathrm{P}<0.001\right)$, suggesting FoxO1 and/or FoxO3A may be associated with Mirk function or kinase activity.

Knockdown of Mirk induces apoptosis involving the downstream signals of FoxO and results in chemosensitivity in vitro. We have reported the concentration- and target-dependent effects on Mirk protein and apoptosis occurred in lung cancer cells induced by Mirk siRNA ( 5-20 nM) and the corresponding individual siRNAs \#1-\#4 of Mirk (8). In this study, we examined the consequence of Mirk knockdown using $20 \mathrm{nM}$ siRNA duplexes \#4 targeting Mirk. We exposed 8 ovarian cancer cell lines to Mirk siRNA for $72 \mathrm{~h}$ followed by assessment of cellular apoptosis. As shown in Fig. 2A, Mirk knockdown by siRNA resulted in cellular apoptosis ( 1.05- to 2.81-fold of control), as evidenced by more Mirk siRNA-treated cells staining with cleaved caspase-3. Intriguingly, the Mirk knockdown-induced apoptosis in variant cell lines appears to be positively correlated with FoxO (FoxO1+FoxO3A) expression. To investigate the mechanisms involved in apoptosis induced by Mirk siRNA, the 


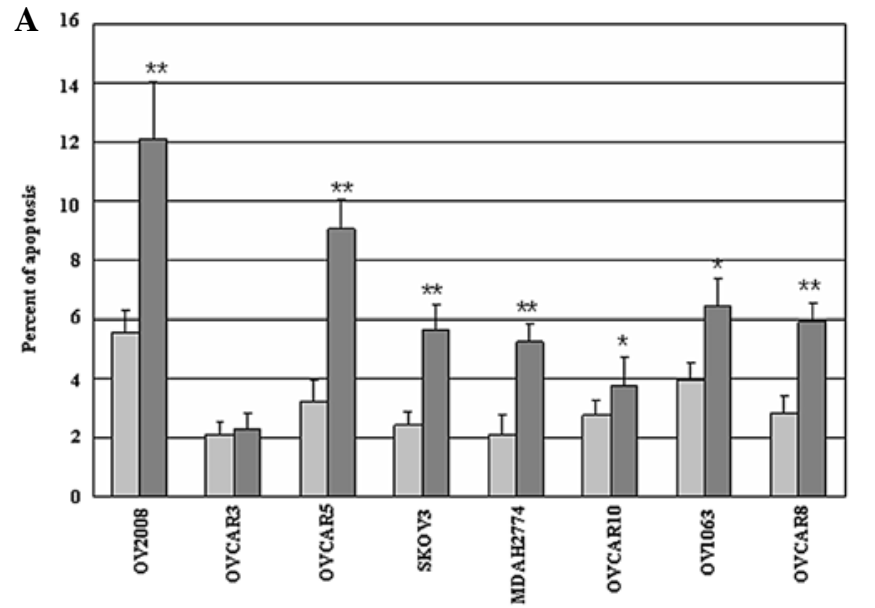

B

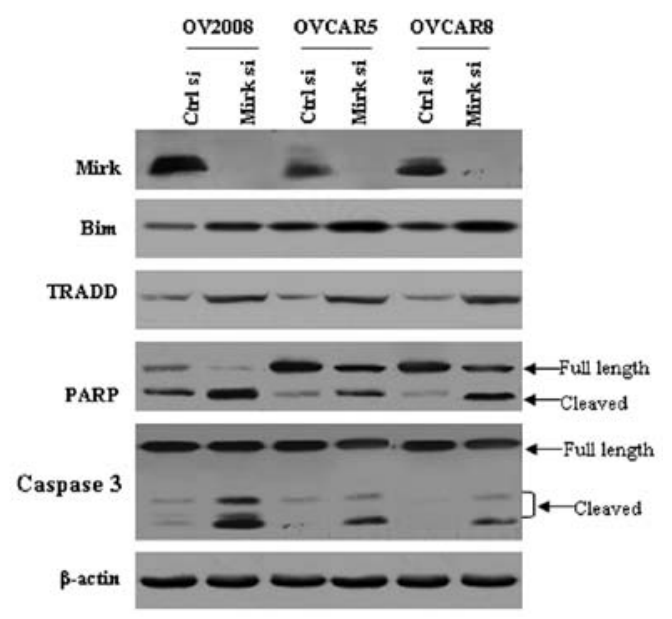

C

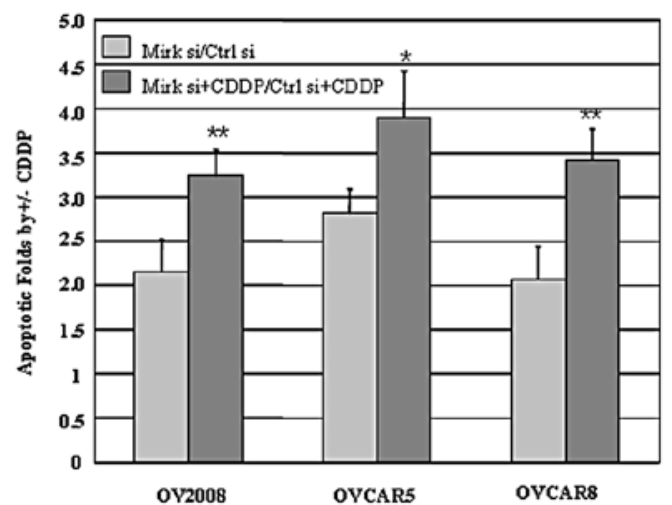

Figure 2. Knockdown of Mirk induces apoptosis involving the downstream signals of FoxO and results in chemosensitivity in ovarian cancer cells. The cells were exposed to siRNAs $(20 \mathrm{nM})$ for $72 \mathrm{~h}$, (A) percentage of cell apoptosis evidenced by positive cells of active caspase- 3 in the cells was assessed by flow cytometry analysis, (B) followed by measurement of Mirk (69 and $71 \mathrm{kDa}$ ), Bim (28 kDa), TRADD (34 kDa), PARP (full length $116 \mathrm{kDa}$ and cleaved $89 \mathrm{kDa}$ ), and caspase-3 (full length $35 \mathrm{kDa}$ and cleaved $17 \mathrm{kDa}$ ) proteins by Western blot analysis with equal loading and transfer shown by repeat probing with $\beta$-actin $(42 \mathrm{kDa})$, and $(\mathrm{C})$ cells were collected and plated in 6-well plates and allowed to grow for $\sim 18-24 \mathrm{~h}$ before 48 -h exposure to $10 \mu \mathrm{M}$ cisplatin, then cell apoptosis (fold of samples by siRNA alone) evidenced by positive cells of active caspase- 3 in the cells was assessed by flow cytometry analysis. ${ }^{*} \mathrm{P}<0.05$; ${ }^{* * *} \mathrm{P}<0.001$ compared with control.

downstream signals of FoxO factors were determined in a representative panel of three ovarian cancer cell lines by Western blot analysis. As shown in Fig. 2B, exposure of these cell lines to Mirk siRNA for $72 \mathrm{~h}$ was associated with knockdown of Mirk, cleavage of caspase-3 and PARP, compared with that shown with control siRNA, and resulted in upregulation of proapoptotic Bim and TRADD in all three cell lines, suggesting knockdown Mirk-induced cellular apoptosis may be associated with FoxO factors as well as their downstream signals. We next investigated the effects of constitutively expressed Mirk on sensitivity of ovarian cancer cells to conventional chemotherapeutics, Mirk siRNA-treated OV2008, OVCAR5, and OVCAR8 cells were exposed to indicated dose of cisplatin for apoptosis assays. Mirk siRNA treatment and exposure to cisplatin in these cells resulted in increased apoptosis (measured in fold) compared with cells treated with control siRNA by caspase-3 assay (Fig. 2C), indicating that knockdown of Mirk sensitizes ovarian cancer cells to chemotherapy-induced apoptosis.

Mirk modulates cell survival associated with nuclear translocation of FoxO. As described above, the phosphorylation of FoxO factors leads to their translocation from the nucleus to the cytoplasm and loss proapoptotic function due to inactivation. Whereas, the unphosphorylated active forms of FoxO reside in the nucleus and induces cellular apoptosis. Previous studies also demonstrate Dyrk1A, the closest isoform of Mirk may phosphorylate FoxO1 and promote nuclear output $(20,21)$, thus there is a possibility that it is the subcellular localization and phosphorylation of FoxO factors but not the total protein level that is altered in Mirk/Dyrk1B siRNA-treated ovarian cancer cells. To explore this hypothesis, both FoxO1 and FoxO3A were detected by immunofluorescent staining in the cell lines OV2008, OVCAR5 or OVCAR8 treated with/without Mirk siRNA. Interestingly, both FoxO1 and FoxO3A are expressed in cytoplasm of these lines (Fig. 3A). Knockdown Mirk induced nuclear translocation of both FoxO1 and FoxO3A in OVCAR5 (Fig. 3B), of FoxO1 alone (not FoxO3A) in OVCAR8 (Fig. 3C), and of FoxO3A alone (not FoxO1) in OV2008 (Fig. 3D). Taken together, these results suggest that FoxO1 and/or FoxO3A may be a novel downstream way in which Mirk serves as an antiapoptotic factor in ovarian cancer cells.

Knockdown FoxO results in less Mirk siRNA-induced apoptosis and decreased sensitivity to chemotherapy in ovarian cancer cells. To further determine the effects of FoxO factors on Mirkmodulated ovarian cancer cell survival, FoxO siRNAs were exposed to the cells of OV2008, OVCAR5 or OVCAR8 treated with/without Mirk siRNA for $72 \mathrm{~h}$, then the apoptotic cells evidenced by cleaved caspase- 3 were detected by flow cytometry analysis. Combined siRNAs of Mirk with FoxO1 and/or FoxO3A led to less cellular apoptosis than Mirk siRNA alone in all three lines (Fig. 4), and less sensitivity to chemotherapeutics (data not shown). These results suggest FoxO1 and/or FoxO3A are involved in Mirk-mediated cell survival in ovarian cancer cells.

Mirk is overexpressed in tumor specimens from clinical ovarian cancer cases. Mirk is expressed at low level in most adult tissues. We examined expression patterns of Mirk in ovarian cancer. As listed in Table I, in this study we not only examined Mirk expression in ovarian cancer specimens included 38 serous and 13 mucinous but also 16 benign cystadenomas and 9 nonneoplastic ovarian cysts by immunohistochemisty. Mirk was 
A
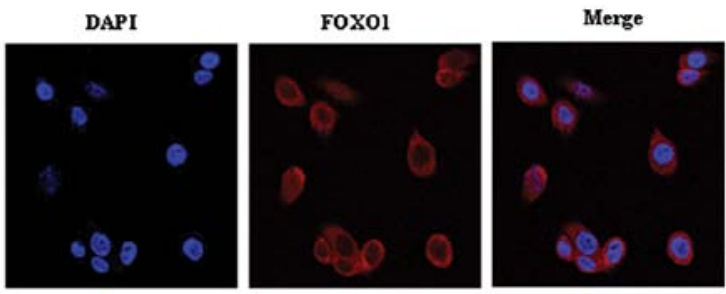

DAPI
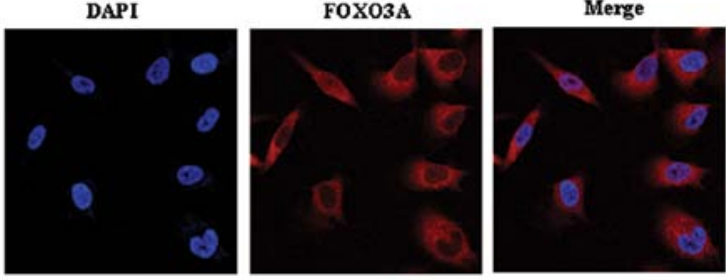

C

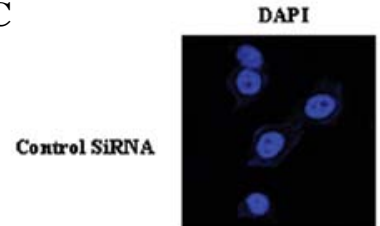

FoXo1
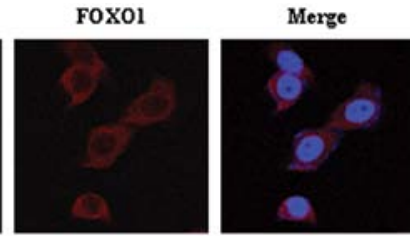

Mirk SiRNA

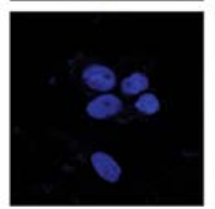

$\mathbf{D}$
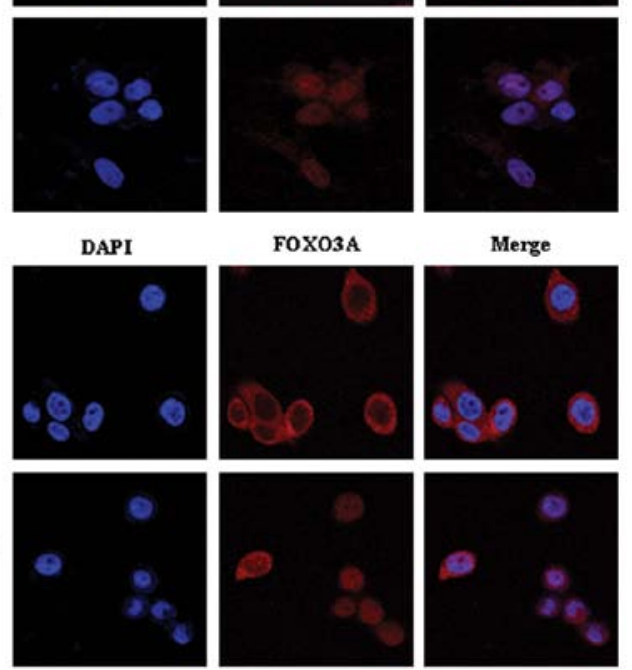

Merge
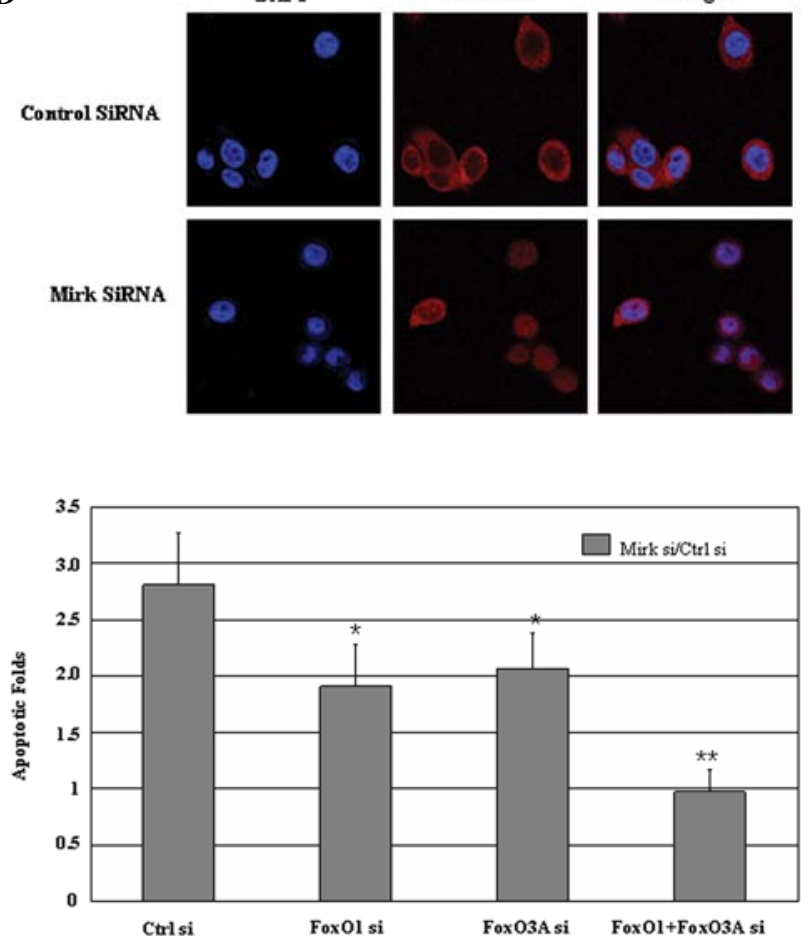

Figure 4. Knockdown FoxO results in less Mirk siRNA-induced apoptosis. The OVCAR5 cells were exposed to siRNAs of FoxO1 and/or FoxO3A combining with/without Mirk siRNA for $72 \mathrm{~h}$, percentage of cell apoptosis evidenced by positive cells of active caspase- 3 in the cells was assessed by flow cytometry analysis. ${ }^{*} \mathrm{P}<0.05 ;{ }^{* *} \mathrm{P}<0.001$ compared with control.

detected in $74.5 \%$ of ovarian cancers and overexpressed in $41 \%$ of the specimens (data not shown), and the incidence was higher than that found in both cystadenomas and non-neoplastic cysts
B
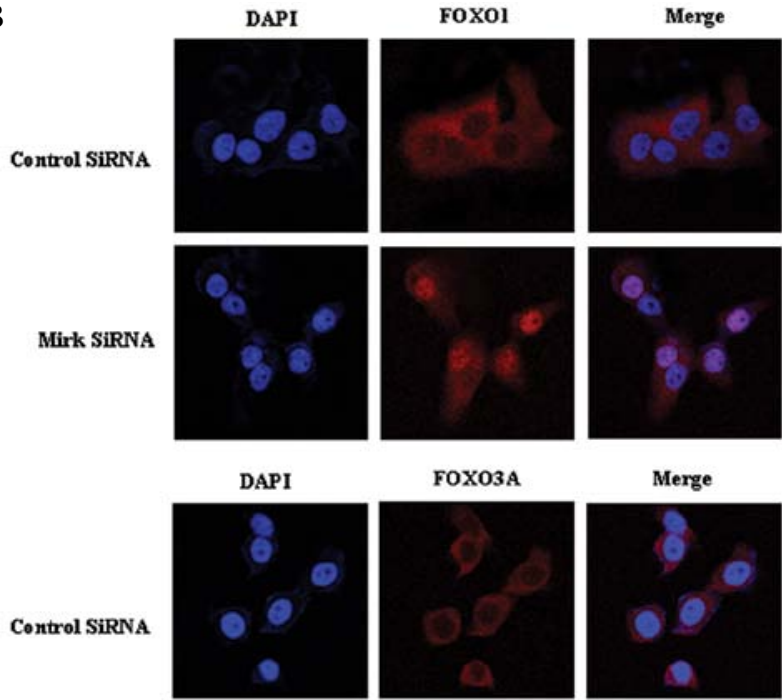

FOX03A
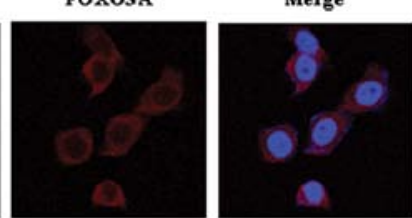

Mirk SiRNA
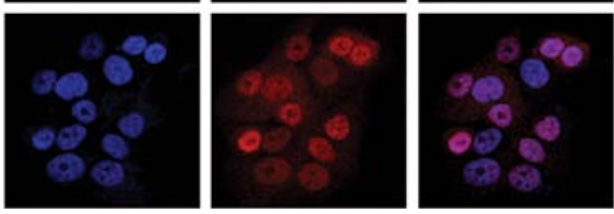

Figure 3. Confocal microscopy of FoxO1 or FoxO3A expression in Mirkmodulated cell survival in ovarian cancer cells. (A), Both panels show the nuclei labeled with 4',6-diamidine-2-phenylindole (DAPI, blue), FoxO1 or Foxo3a was cytoplasmic visualized with the use of Alexa 594 (red), and the merge of DAPI and FoxO1 or FoxO3A, respectively. (B), Upper panels show DAPI, FoxO1, and the merge of DAPI and FoxO1 with/without Mirk knockdown in OVCAR5 cells. Bottom panels show DAPI, FoxO3A, and the merge of DAPI and FoxO3A with/without Mirk knockdown in OVCAR5 cells. (C), Both panels show DAPI, FoxO1, and the merge of DAPI and FoxO1 with/ without Mirk knockdown in OVCAR8 cells. (D), Both panels show DAPI, FoxO1, and the merge of DAPI and FoxO3A with/without Mirk knockdown in OV2008 cells.
A

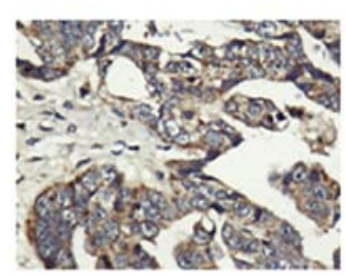

C

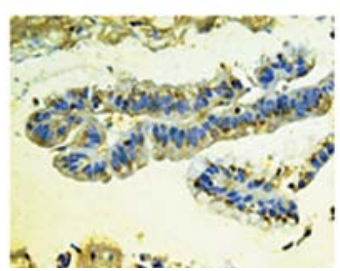

B

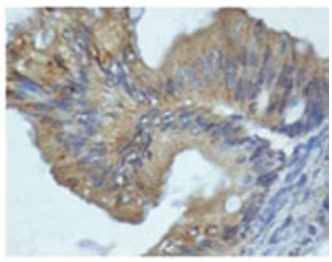

D

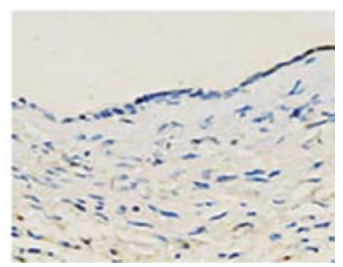

Figure 5. Mirk expression in ovarian cancer specimens and non-malignant ovarian tumors or cysts. The panels show immunohistological Mirk expression in formaldehyde-fixed and paraffin-embedded histologic sections of serous cystadenocarcinoma (A), mucinous cystadenocarcinoma (B), cystadenomas (C), and non-neoplastic ovarian cysts (D). The Mirk is cytoplasmic and the nuclei were counterstained with hematoxylin (original magnification x400). 
( $\mathrm{P}<0.001$ and $\mathrm{P}<0.05$, respectively). We further found the overexpressed Mirk was located in the cytoplasm of ovarian cancer specimens (Fig. 5A and B) similarly to the findings in other organ cancers $(4,22)$. Compared with Mirk expression in most of ovarian cancer specimens, it was weakly expressed only in about $37 \%$ ovarian cystadenomas (Fig. 5C) and not expressed in the ovarian non-neoplastic cysts (Fig. 5D), indicating Mirk may be associated with ovarian tumorigenesis.

\section{Discussion}

Our study demonstrates that Mirk/Dyrk1B is overexpressed in a variety of ovarian cancer cells and clinical specimens. Knockdown of Mirk/Dyrk1B induced apoptosis of ovarian cancer cells in vitro and sensitized ovarian cancer cells to chemotherapeutics. These results are consistent with previous studies (3-6,8) on other types of human cancers, indicating that Mirk may be a novel therapeutic target for ovarian cancer treatment. Furthermore, we found Mirk to be expressed at higher levels in both serous and mucinous ovarian cancers than that in cystadenomas and ovarian non-neoplastic cysts. Therefore, our study along with others $(23,28)$ suggests that Mirk may also play a role in ovarian tumorigenesis.

To date, the downstream signals of Mirk remain unclear. Given the main function of Mirk in ovarian cancer cells in mediating cell survival observed in this study, the mechanisms involved may include FoxO family members, such as FoxO1 and/or FoxO3A as well as their downstream signals, which are constitutively expressed in ovarian cancer cells (Fig. 1A). To the best of our knowledge, our study is the first to show an effect of FoxO involved in Mirk-mediated cancer cell survival. It has been reported that Dyrk1A, the closest family member to Dyrk1B/ Mirk, may phosphorylate FoxO1 at ser329, a novel in vivo phosphorylation site (25), and promote the phosphorylated FoxO1 nuclear output $(20,21)$. The knockdown of endogenous Dyrk1A by siRNA mediates cellular localization of FoxO1 in immortalized cells (24). Thus, we hypothesize that FoxO factors may contain a Mirk phosphorylation site, on which further study is needed. In our study, we also found that FoxO1 and/or FoxO3A nuclear translocation is concomitant with cell apoptosis induced by Mirk knockdown, which together with previous studies $(24,25)$ suggest that it might be the subcellular localization and phosphorylation of FoxO that is altered in Mirk siRNA-treated ovarian cancer cells.

Taken together, Mirk/Dyrk1B is overexpressed in a wide spectrum of ovarian cancer cell lines and human specimens. The Mirk/Dyrk1B-mediated cell survival in ovarian cancer cells is associated with FoxO subcellular localization. Therefore, Mirk/Dyrk1B may be a novel target for treatment of ovarian cancer.

\section{Acknowledgments}

We thank Dr Yuyan Zhu (University of South Florida Medical College, Tampa, FL, USA), for helpful discussions as well as English editing. This work was supported in part by the National Natural Science Foundation of China (81172457 to J.G.) and Natural Science Foundation of Fujian Province, P.R. China (2010J01236 to X.Y.). The authors declare no conflicts of interest.

\section{References}

1. Ozols RF, Bookman MA, Connolly DC, et al: Focus on epithelial ovarian cancer. Cancer Cell 5: 19-24, 2004.

2. Becker W and Joost HG: Structural and functional characteristics of Dyrk, a novel subfamily of protein kinases with dual specificity. Prog Nucleic Acid Res Mol Biol 62: 1-17, 1999.

3. Lee K, Deng X and Friedman E: Mirk protein kinase is a mitogenactivated protein kinase substrate that mediates survival of colon cancer cells. Cancer Res 60: 3631-3637, 2000.

4. Mercer SE, Ewton DZ, Shah S, Naqvi A and Friedman E: Mirk/ Dyrk1b mediates cell survival in rhabdomyosarcomas. Cancer Res 66: 5143-5150, 2006.

5. Yang C, Ji D, Weinstein EJ, et al: The kinase Mirk is a potential therapeutic target in osteosarcoma. Carcinogenesis 31: 552-558, 2010.

6. Deng X, Ewton DZ, Li S, Naqvi A, Mercer SE, Landas S and Friedman E: The kinase Mirk/Dyrk1B mediates cell survival in pancreatic ductal adenocarcinoma. Cancer Res 66: 4149-4158, 2006.

7. MacKeigan JP, Murphy LO and Blenis J: Sensitized RNAi screen of human kinases and phosphatases identifies new regulators of apoptosis and chemoresistance. Nat Cell Biol 7: 591-600, 2005.

8. Gao J, Zheng Z, Rawal B, Schell MJ, Bepler G and Haura EB: Mirk/Dyrk1B, a novel therapeutic target, mediates cell survival in non-small cell lung cancer cells. Cancer Biol Ther 8: 1671-1679, 2009.

9. Kops GJ, De Ruiter ND, De Vries-Smits AM, Powell DR, Bos JL and Burgering BM: Direct control of the Forkhead transcription factor AFX by protein kinase B. Nature 398: 630-634, 1999.

10. Nemoto S, Fergusson MM and Finkel T: Nutrient availability regulates SIRT1 through a forkhead-dependent pathway. Science 306: 2105-2108, 2004.

11. Fu W, Ma Q, Chen L, et al: MDM2 acts downstream of $\mathrm{p} 53$ as an E3 ligase to promote FOXO ubiquitination and degradation. J Biol Chem 284: 13987-14000, 2009.

12. Yang Y, Hou H, Haller EM, Nicosia SV and Bai W: Suppression of FOXO1 activity by FHL2 through SIRT1-mediated deacetylation. EMBO J 24: 1021-1032, 2005.

13. Brunet A, Bonni A, Zigmond MJ, et al: Akt promotes cell survival by phosphorylating and inhibiting a Forkhead transcription factor. Cell 96: 857-868, 1999.

14. Nakae J, Barr V and Accili D: Differential regulation of gene expression by insulin and IGF-1 receptors correlates with phosphorylation of a single amino acid residue in the forkhead transcription factor FKHR. EMBO J 19: 989-996, 2000.

15. Dijkers PF, Medema RH, Lammers JW, Koenderman L and Coffer PJ: Expression of the pro-apoptotic Bcl-2 family member Bim is regulated by the forkhead transcription factor FKHR-L1. Curr Biol 10: 1201-1204, 2000.

16. Dijkers PF, Medema RH, Pals C, et al: Forkhead transcription factor FKHR-L1 modulates cytokine-dependent transcriptional regulation of p27(KIP1). Mol Cell Biol 20: 9138-9148, 2000.

17. Deng R, Tang J, Xie BF, Feng GK, Huang YH, Liu ZC and Zhu XF: SYUNZ-16, a newly synthesized alkannin derivative, induces tumor cells apoptosis and suppresses tumor growth through inhibition of $\mathrm{PKB} / \mathrm{AKT}$ kinase activity and blockade of AKT/FOXO signal pathway. Int J Cancer 127: 220-229, 2010.

18. Skurk C, Maatz H, Kim HS, Yang J, Abid MR, Aird WC and Walsh K: The Akt-regulated forkhead transcription factor FOXO3a controls endothelial cell viability through modulation of the caspase-8 inhibitor FLIP. J Biol Chem 279: 1513-1525, 2004.

19. Tang TT, Dowbenko D, Jackson A, Toney L, Lewin DA, Dent AL and Lasky LA: The forkhead transcription factor AFX activates apoptosis by induction of the BCL- 6 transcriptional repressor. J Biol Chem 277: 14255-14265, 2002.

20. Arimoto-Ishida E, Ohmichi M, Mabuchi S, et al: Inhibition of phosphorylation of a forkhead transcription factor sensitizes human ovarian cancer cells to cisplatin. Endocrinology 145: 2014-2022, 2004.

21. Goto T, Takano M, Hirata J and Tsuda H: The involvement of FOXO1 in cytotoxic stress and drug-resistance induced by paclitaxel in ovarian cancers. Br J Cancer 98: 1068-1075, 2008.

22. Deng X, Ewton DZ and Friedman E: Mirk/Dyrk1B maintains the viability of quiescent pancreatic cancer cells by reducing levels of reactive oxygen species. Cancer Res 69: 3317-3324, 2009. 
23. Hu J and Friedman E: Depleting Mirk kinase increases cisplatin toxicity in ovarian cancer cells. Genes Cancer 1: 803-811, 2010.

24. Chang $\mathrm{HS}$, Lin $\mathrm{CH}$, Yang $\mathrm{CH}$, et al: Increased expression of Dyrk la in HPV16 immortalized keratinocytes enable evasion of apoptosis. Int J Cancer 120: 2377-2385, 2007.

25. Von Groote-Bidlingmaier F, Schmoll D, Orth HM, Joost HG, Becker W and Barthel A: DYRK1 is a co-activator of FKHR (FOXO1a)-dependent glucose-6-phosphatase gene expression. Biochem Biophys Res Commun 300: 764-769, 2003.

26. $\mathrm{Hu} \mathrm{J}$, Nakhla $\mathrm{H}$ and Friedman E: Transient arrest in a quiescent state allows ovarian cancer cells to survive suboptimal growth conditions and is mediated by both Mirk/dyrk1b and p130/Rb2. Int J Cancer 129: 307-318, 2011.
27. Chakrabarty A, Sanchez V, Kuba MG, Rinehart C and Arteaga CL: Breast cancer special feature: feedback upregulation of HER3 (ErbB3) expression and activity attenuates antitumor effect of PI3K inhibitors. Proc Natl Acad Sci USA 22: 5021-5026, 2011.

28. Thompson FH, Nelson MA, Trent JM, et al: Amplification of 19q13.1-q13.2 sequences in ovarian cancer. G-band, FISH, and molecular studies. Cancer Genet Cytogenet 87: 55-62, 1996. 